Published in final edited form as:

Neurotoxicol Teratol. 2011 ; 33(6): 607. doi:10.1016/j.ntt.2011.10.005.

\title{
Introduction to zebrafish: Current discoveries and emerging technologies for neurobehavioral toxicology and teratology
}

\author{
Edward D. Levin ${ }^{a}$ and Robert L. Tanguay ${ }^{b}$ \\ aDepartment of Psychiatry and Behavioral Sciences, Duke University Medical Center, USA \\ ${ }^{\text {b}}$ Department of Environmental and Molecular Toxicology, Oregon State University, USA
}

This special issue of Neurotoxicology and Teratology "Zebrafish: Current Discoveries and Emerging Technologies" brings together the latest research concerning the use of zebrafish models for screening and mechanistic studies in neurobehavioral toxicology. The goal is not only to collect, in one place, the cutting-edge research findings in neurotoxicology obtained with the zebrafish model, but also to provide reviews of the key methods needed to the use of zebrafish for future neurotoxicological research.

The zebrafish (Danio rerio) model is rapidly maturing into a major platform for investigating the molecular and cellular mechanisms of developmental neurobehavioral toxicology, and for increasing the throughput for screening large numbers of chemicals for developmental neurotoxicity. In 2004, Neurotoxicology and Teratology published a special issue, recognizing the promise of the zebrafish model for neurotoxicology research. In the ensuing seven years, zebrafish have proven useful in the study of the developmental aspects of neurotoxicology. Important recent advances in this field have been achieved in making another special zebrafish issue timely.

Zebrafish are now well-established as an outstanding complementary model for the study of developmental biology because of their fecundity and the transparency of the larvae, which enable researchers to visualize morphological changes throughout embryonic development. Transgenic reporter systems can mark particular cell types allowing non-invasive tracking during this period. Antisense morpholinos (MOs) can be used to transiently suppress gene expression and thereby modulate selected molecular events during early development. With the readily available zebrafish genome sequence, the role of any gene product in developmental neurobehavioral toxicology can be elucidated.

Zebrafish are particularly useful for studying development of the nervous system. For developmental neurotoxicology, the impact of toxicants can be assessed from the mechanisms of their entry into cellular processes, through the disturbances of integration of intra- and inter-cellular signaling, to consequent behavioral impairments.

Whereas elegant tools have been created to study molecular and cellular mechanisms in zebrafish, meaningful, sensitive, reliable and efficient paradigms for behavioral assessment are needed to determine the functional impacts of toxicant exposure in this model. Zebrafish and other non-mammalian organisms including medaka, killifish, goldfish, drosophila, 
honeybees and the more elementary $\mathrm{C}$. elegans can serve as powerful complementary models for classic in vitro and in vivo mammalian models.

The first section of this special issue consists of articles that provide overviews and discussions of important methodological considerations for use of zebrafish in neurotoxicological research. Included is an overview of the rationale and advantages of the zebrafish model and critical considerations of how such studies can usefully be conducted.

Note that zebrafish are not the only fish species used for neurobehavioral studies. There is a long history of research with other species, particularly goldfish (Carassius auratus); therefore, an article using goldfish is included for comparison.

The second section of the special issue includes articles describing research on effects of therapeutic drugs including lithium, ketamine, methylphenidate, and valproate. In addition, the impact of consumer drugs and drugs of abuse like caffeine and ethanol is represented in this issue. Some of these drugs have relatively selective effects on particular receptor and transporter systems so that the research not only sheds light on the potential neurotoxic risk posed by these drugs, but also helps elucidate critical neural mechanisms underlying susceptibility to neurotoxicants.

The third section of the special issue contains articles focused on the neurotoxicity of environmental contaminants including metals such as mercury, lead, tin and silver nanoparticles. The impact of other neurotoxic contaminants including chlorpyrifos and 4nonylphenol is also explored. Many of these environmental toxicants have extensive bodies of research conducted using in vitro and mammalian models and associated epidemiology. With this rich literature background, the studies in this issue serve as excellent examples of how studying neurotoxicity in the zebrafish model can substantively add to this spectrum of complementary models. Zebrafish fill an important gap in the spectrum, having complex brains whose physical development can be examined in real time, and then tested for behavioral function at an appropriate age.

In conclusion, zebrafish provide a useful model particularly for developmental neurotoxicology studies. The studies presented in this special issue of Neurotoxicology and Teratology provide numerous examples of how the zebrafish not only serve as a complementary intermediate model between cell based in vitrosystems and the classic mammalian models, but they also offer unique advantages that can be exploited to more rapidly unravel mechanisms of neurotoxicity. 